\title{
INY IJYY- MY
}

Kaorewygoo Tapirapé ${ }^{1}$

\section{RYBÈBÙTE}

Riu bdedyynana waõmysydyyna, iny irbi tuhuti rusare, taikimy tahè retere idi araõmysydyynykremy isby iny rierykremy timybo juhu hyyna mahadu rariunymy ryiramyhy-my, mykimy tahè iny ierydu mahadu isiko reijyydyynyrenyre riu timybo juhu hyyna mahadu rariunyryiramyhy-my, sõèmy ièrydu mahadu riwawiòhenanyrènyre.

PALAVRAS-CHAVE: Iny Òlòna-my ijyy. Riu-my ijyy. Wyhy. Ohote. Waxiwahate. Bdedyynana.

\section{RESUMO}

O tema do meu projeto extraescolar é Riù. Hoje são poucas usadas as técnicas e as armas na caçada, e por isso escolhi esse tema para trabalhar. Fiz as pesquisas, buscando as informações sobre os conhecimentos que estão sendo desvalorizados pelos jovens de hoje. Com ajuda dos entrevistados, aprendi através do meu trabalho as regras que se utilizavam desse conhecimento. Espero que as pessoas tenham prática novamente para seguir as regras dos seus antepassados.

PALAVRAS-CHAVE: Origens do povo. Caçada. Flecha. Borduna. Arco. Conhecimentos.

\section{ABSTRACT}

The theme of my out-of-school project is Riù, today there is little use of techniques and weapons in the hunt, so I chose this theme for me to work on, I researched the information about knowledge that is being devalued by today's youth, with the help of the interviewees I learned the rules that were used of this knowledge through my work, I hope people practice again and follow the rules of their ancestor.

KEYWORDS: Origins of the people. Hunt. Arrow. Borduna. Arch. Knowledge.

1 Licenciado no Curso de Educação Intercultural (Ciências da Cultura) pela Universidade Federal de Goiás. Professor da Educação Básica. Escola Estadual Indígena Hawalòra. Pesquisador da Ação "Saberes Indígenas na Escola", Rede UFG/UFT/UFMA. Aldeia Hawalòra, MT, Brasil. E-mail: kaorewygoo.tapirape@gmail.com. 


\section{Introdução}



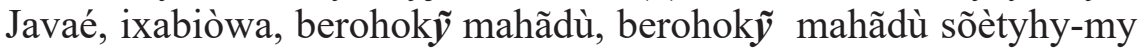
roimyhỹre, Mato Grosso ki, Goiás, Tocantins, Pará, kia estado ki


mahãdù rariunymyhỹre.

Kaa waõmysỹdỹỹna extraescolar riu bdèdỹỹnana tai awimy rahare, tai tahè retere butuna dèè tako-ò riwyrènykremy, rièryrènykre-my, timybo iny rexiywinymyhỹre riu-my, kaa waõmysỹdỹỹna he awi rarè, Tori dèè rièryrènykre-my timybo iny rariunymyhỹre rièryrènykre-my. Jiarỹ kaa watyyrtina waèryna ariwinykre-my tako-ò, ariijekre-my juhu mahãdù ijyy adiwykre-my ièryna rèny hetxina, kiè-my wijina mahãdù riuheõmyhỹre, kiarbi tahè relekre juhu mahãdù èryna tby, rièryrènykre-my isby wijina mahãdù.

\section{Resumo}

Kaa waõmysỹdỹỹna extraescolar ki ariteòsinykre aòherekibo inydèèrèny iny-my awire riu bdèdỹỹnana-my, timybo juhu iny hỹỹna mahãdù riu-my èlèèlè-my rirahùmyhỹre, timybo rexiywimyhỹrenyre riu-ò inylsina-u.

\section{Referência teórica}

Kaa waõmysỹdỹỹna ki aõbo aonityhy-my rauhèmyhỹre riuki raèrykre-my, riudù mahãdù hèmy ramy rètèhè-my raèrykre-my timybo riudù mahãdù rariunymyhỹre, tibdeòbo rirahùmyhỹre riu-my. Kaa waõmysỹdỹỹna retere tkièmy rèèrybinare juhu mahãdù timybo rariunymy rãiramyhỹ, timybo rexiywinymyhỹrenyre riu-ò, inyumy mahãdù wna rèwinyre tii bòhò wako relyyrènyre riu bdèdỹỹnanamy timybo juhu hyyna mahãdù rariuny-my rãiramyhỹ. Iny dèè awire isby raèrykre-my timybo juhu mahãdù rariuny-my rãiramyhỹ rusakèlau juhu mahãdù èryna, rauhèhyy rarekre-my wijina mahãdùmy, iny bdèdỹỹnana-u iwysemy tahè wijina mahãdù rièrytyhykremy riu bdèdỹ̃̃nana. 


\section{Metodologia}

Inatxi (2) hãwã-ò reijere ijyydù Hãwãlòra-ò, Santa Terezinha, Mato Grosso ki roimyhỹ, Buridina-ò, Aruanã, Goiás ki roimyhỹ, Dezembro 2015 ú, Agosto/2016 ú, Buridina-ki. Riu-ù riudù mahãdù sohoji sohoji rètyyraxiny myhỹ, arirtinykre-my timybo iny rariunymyhỹre-my, dyyraxina-ù tahè delyymyhỹdenyde aõtyhytyhybo riu-ki rùrùmyhỹre, inatao(3) riu-my rare jyrè mahãdù hè-my.

\section{Iny Òlòna-my Ijyy}

Juhu rki hèka iny bèrahatxiwo-my rasỹnymy rãiramyhỹ. Ruu sohoji-my, inataõ iny rki rùrù-my txuu idi rèamyhỹre. Iwèdùrèny tamyreny rarybèmy raremyhỹ, sùù-my rarunymyhỹrènykè-my. Tarkihè ijoi mahãdù raubtymyhỹrenyre ijõ rki irarù-ò rèarènyre, ibràra-ò, beà-ò, ibòò-ò rki. Iroorèny-my Tarkihè sohoji ibòò-ò rèare. Tarkihè ryy itxiriè-my roiredile Tarkihè ralòmy rèare. Ryiremy kiè rki kawse hãlùù ijò mna wotxi.

- "Idi knyini aralò aõhebo arutyynykrelemy".

Idi rkihe ralò-my rare, ralò-my rara hkyhky Tarkihè rexidoruny-my rèare. Kia Tarkihè kabdè roire, ynyra, nawii rexiyjany-my roimyhỹ, kia Tarkihè rètèhèmy runyra.

"Be! Aõherewe bdè wihikỹ-txi rèara". Kia Tarkihè rètèhèmy mna tyretyredi rare, rarahkyhky Tarkihè sùù-ò rèhèmnyre.

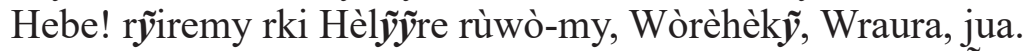
Ityti tyhy-ò rèhèmnyra-u Tarkihè Hãlòè-le rariamy rare, Bròre, Ôri, Uwè. Ahù ijà ldu he rare, rara hkyhky tarki Ixy ijoi wna le witxi rèare, rariamy rara hkyhky kuatxi rèamy Tarkihè bdèraty-txi rèare, Urà, Samõ, Horeni, ixby tako-ò rariara-u tarki ynyra-le roire.

Hèèbe! Bero Tarkihè roire.” Aõherewe sòwe bdèwi”. Ynyra-ò rùnyre, ruri rehe rki riwinyre, tamy bdèraty-sò rihynykre-my, èlèèlèmy rkihè rihynymyhỹ bdèraty-sò.

"Ariwykreini"

Myy rkihè rexirybènyre. Itxèrèna-my riwykre-my, ibdè-ki ijõdire hãrele tarki riwyre, bdèraty-sò òtaòta sõèwe-my. Idi Tarkihè nade tku wo-ò, ijoi ko-ki ralore mahãdù rki roire rexiyjany-my, tamyreny riwahinyre.

"Ijoi rèny! Aõkõre inihè wabdèsamy inihe rare", rawyhyrarònymy rkihè rare. 
"Bètèhèbèny ini bdèraty-sò rèwyreri-ò". Kia tarki urile riruidỹỹnyre, "Taruiteri! Kaa bdèraty-sò kamy rỹirale tèwytèri ùrile jiarỹ boho biwawasarbuny bènykemy òsyny tarùtenytèri”, myle rki tamy rarybèrènyre, tai Tarkihè ùrile rabdèwore. Rare tarki bdèratydi tawèdù-ò, tarki tule tamy rarybere ijoi mahãdù rybè yrbule, urile rarùirerile-my.

"Aõkõrehe inatàhà-my ãdèè rarybè reri, kaa tukõ keki ta ãdèè anakè aõkõ rare. "Bdè awi hèka katxi doide".

Ixby rki tadinõdù rèny-ò rarybère.

"Tùu ijoi taditeranymyhỹde-ki hèka iwahimy ãdèè rarèri, tukõ keki ta tiu ãdèè iwahimy anakè aõkõ rare".

Myki Tarkihè, riwoijaranyre tadinõdurèny.

"Hebose aratyhynykreini, ta urile mykre, iny dèbò sohoji bidykre ixihè-my. Tai awimy ijyy-di bditèhèmnybènykre-my awire aõbo?".

Hitxi rkihè rexiywinymy rekoa, roire hkyhky tarki rèhèmnyrènyre hãlùù ijò-ò idirèny.

"Rkòireny bdewo rènyõmyta rikiòlònyrènykre". Tarkihè riòlònyrènyre raworèrènyra Tarkihè ihèmy rara mahãdù, ynyra-ò rki idirèny rùbehere. "Kaò inihè inywèdurèny kdèòbykre". Rkòirèny tahè.

Tarkihè rèhèmnyrènyre hãwã-ò, hitxi rki dirahu ijoi mahãdù tamyrèny rarybère rùidùleheky ixihèmy ridyre-my. Tai Tarkihè tki boho tuu robirènyramy relyymy ratxirènyreri, idi wisdyky-di txuu ralore. Bdèsò-my Tarkihè tamyrèny rarybère.

"Mykre inihè ãdèèrèny ararybekre, iSinamy Bòibènykre ibutumy ijoi rèny. jiarỹ le ta ãrãrikre hãwyy-di itxèrèdù-my. Myy rkihè taijoi rènydè rarybère. Taita rkihè ijo biurasòmy rki rirahùre tamy itxèrèmy, ijoi Tarkihè ròhònyra tasõè-di kabdè-ò, idi Tarkihè rètèhèmy roire, kaò rkòirènykre itxèrè-my". Bdèrahy-di rkihè ririranymy ratxirènyrèri, rarahkyhky Tarkihè rùbèhèrènyra bèra ijà-ò kianihè ihyde, utura, òtuni rki robirènyra, ixy, hàlà tule rki tùù sõèmy rỹi reri. Tarkihè tamyrèny rarybère.

"Bètèhèbèny ta inihè, tiwarùidỹỹnymyhỹtènyte wahe. Tii mahãdù rarybère.

"Inatàhàmyhè tuu wadèèrèny tarybètèri,aõkõre syna raholarènyre inatàhà-my bohe tadelyymyhỹtenyte. Kaini iny wèdùrèny rarerikre ixybyle, Rkòirèny tahè". 
Ròireri hkyhky tarki ixy-ò ditèhèmnyde, ijoi rabdèsa-my dèhèmnydenyde. Ijoina-ki Tarkihè tùù relyyrènyre, tai tarki tawèdu reny-ò relyyrènyre, tai tarki iwèdureny narybede.

"Heboxe rkòirenykre, iny-õ rarikre hãwyy-di itxèrèdù-my, jiarỹ tahè retxiõnyreri-ki arakre tulobixi-my".

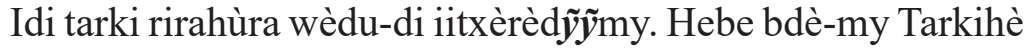
raworèra.

"Inatàhàkyle-my kòri mama wako tadelyymyhỹtenyte, rùisana ãdoholamyhỹde, kahè reijèwahare kawsè, tai rki ru-my bdè riijerènyre. Tule rkiawimy tai rahare, urileta maaõmys $\tilde{y} \mathrm{~d} \tilde{y} \tilde{y}$ nybenykre.

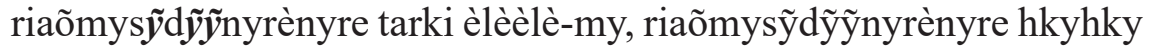
Tarkihè taõmysỹdỹỹna rahurènyre, titxibo inataõ txuu inaubiòwa-di òsyny rihurènyre.

Ròhònyra Tarkihè ixy mahãdù awi-my rki tai raharènyra. Tasỹrèny riywiny-my ryireri sõèmy, rèamy Tarkihè tamy rỹireri. Tarki tamyrèny rarybera.

"Aõkõre hèka katxi weryry hãwãna-ki rèamy doimyhỹde awi hèka rare hãwãna-ki. Taita rkihè dòidènyde, ityamy rkihè tki kdòhònykre-my namyhỹde Iwèdùrèny, delemny rkihè ijõ ròhònyra tule hãwã-ki awimy raharènyra, tkiè hky tararù ridèrènyreri idinodùrèny ròhònyreri kia Tarkihè iwè kaa rare kyboi rki inire. Ijõ Tarkihè ihewo-ki rarirèri mahãdù kdòhònydènykre-my ròirènyreri tawèdùrèny hèmy, kiata rkihè aõkõhykyre tkiè-my Iwèdùrèny ròhònyõhykyre, iwenihiky reki wahè. Òlòmy Iwèdùrèny ramyhỹre rki katele-my ròhònymyhỹre. Biu-ò rki hèka ròhònymyhỹre iumy, bde-ò Tarkihè urile reàrenymyhỹre, uritrè rki òlòmy riwisnymyhỹre Tarkihè iwo rexièryre ixirbi, Tarkihè taijoireny dèè rarybere,

"Aròrònyõkreinyhe, kiatxi was ỹ ròhònymy ramyhỹre mahãdù ixybyle kdòòsèdènykre, ijõ mahãdù tahè kaki-le reokròmy watxireri. Myy Tarkihè naõhykydenyde ixybyle rki riòksèrènyre, hewo-ki rarire mahãdù wana. Kia-my rki hèka iny ròhònymy kabdè-ò roimyhỹre.

\section{Riu-my ijyy}

Hãbu le hèka riu-my ramyhỹre, juhu hèka irodu dè-di rỹiramyhỹ, iny berohokỹ mahãdù riu riuhèmyhỹre inatxi-my, èlè̀lè riu-my iny rawna rki inyinataõ-my ramyhỹre oworu-my aõi tki ijodi tyhy wna, iny sõèmy riu Tarkihè wii riwirarèamyhỹre, ijõ rkihè iròdù riraomyhỹre titxibo ibèmomona-ò, dè tikibo sõèmy iwary tmyramy 
robi rènyrawna tai riraòmyhỹrenyre, bdèraty sõ sõwèki tule riudu mahãdù riijemyhỹre iròdù. Kaa riu-my rirahùmyhỹre ijasò dò-my kia he rki butunahakỹ-my rirahùmyhỹre bjiu-txi,bdero-txi,aotxile iròdùtxi rèakre-my kiamy rki riu iny sõèhek $\tilde{y}$-my riwinymyhỹre rare, riu na rkihè biu õ-u awire iny dèè kièmy rkihè iròdù iu sõèmy rỹirèrimyhỹre bdèro-my,bdèbira-my,bjiu-my irutaõ-my, iu rkihè iròdù rexijuamy rỹirèrimyhỹre, biu ranananymyhỹre-u rkihè riu aõhètyhyõrè, iròdù riu-ù rirbunymyhỹrenyre , õritxi, hãju, hãuri, otubuna, juhu hỹỹna-u waxiwahatè, wyhy, òhòtè, tõnori, myy riuhemy rỹiramyhỹ riu-ù, wiji

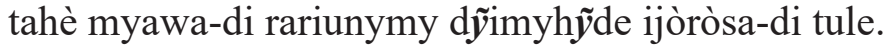

\section{Ijaso osoro-my ijyy}

Hỹỹna mahãdù tahè ijaso riòsùhomyhỹre-u ibùtù-my jyrè

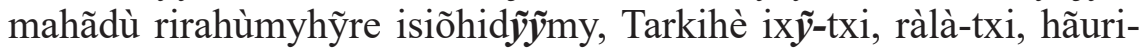
txi, õritxi-txi, rèamyhỹrenyre riu-ki, Tarkihè idi dirahùmyhỹte worysy ryryhyky-my bdèrahy rbi, ijoina rbi Tarkihè riwymyhỹre jyrè mahãdù ijasò se-ò iròdù dedi.

\section{Tarỹy-u riu-my ijyy}

Kaa ijasò raryyny myhỹre-di rexiõhinymyhỹre-u Tarkihè iwitxira-my idi rirahùmyhỹre, idi tỹhi-my titxi rirahùmyhỹrenyre, juhu hỹỹna bdè-u rkihè sõèmy ixỹy , ràlà, utura, otuni rỹiramyhỹ, jyrè mahãdù rkihèrirahùmyhỹreibùtùmy isiõhidỹỹmy, ibùtùmy rkihèijasò

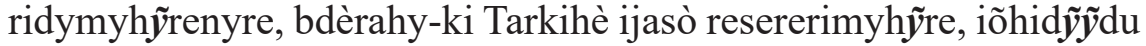
hitxi-my rỹirèrimyhỹre ix $\tilde{y}$, ràlà rijemy, kaa ijasò rèsèrèrimyhỹre

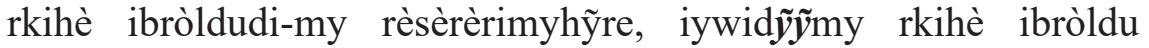

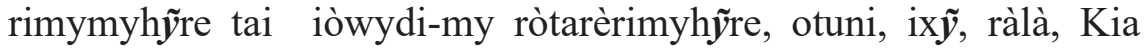
rkihè ritarèrimyhỹre, hãwã-ki hãwyy rèsèrèrimyhỹre yrbù-lè, hèradù

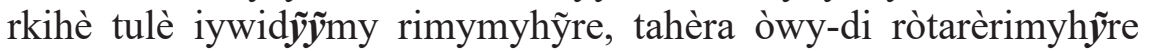
tulè ijasò bròldu wsèlè isy, ràlà, otuni rkihè ritarèrimyhỹre, kaa sõèmy rèèrynymyhỹrenyre-u Tarkihè widèè rarybèmyhỹrenyre ijoihè raa ,ijoi raa my Tarkihè dirahùmyhỹde, ruwètya rkihè rèhèmnymyhỹrenyre hãwã-ò iny tỹhilemy rkihè wii rotymyhỹre, ijasò ijoina-ki rawinymyhỹre-u hkylè, Tarkihè iny mahãdù rexixamyhỹre, kie-my rkihè tadò-di relòlòi-my rỹirèrimyhỹre, dèbòitùè txu waaitùè txu bdè rkihè riwymyhỹrenyre riu-ki. 
Foto 1 - Juhu rki ijoi riu-my rirahùkreko-ki raxiwèmyhỹre tyytby-ò, lateni-ò, txyreheni-ò kia boho dèè rkihè aònityhy rexitòènymyhỹre, kie-my rkihè tii boho aònityhyre.

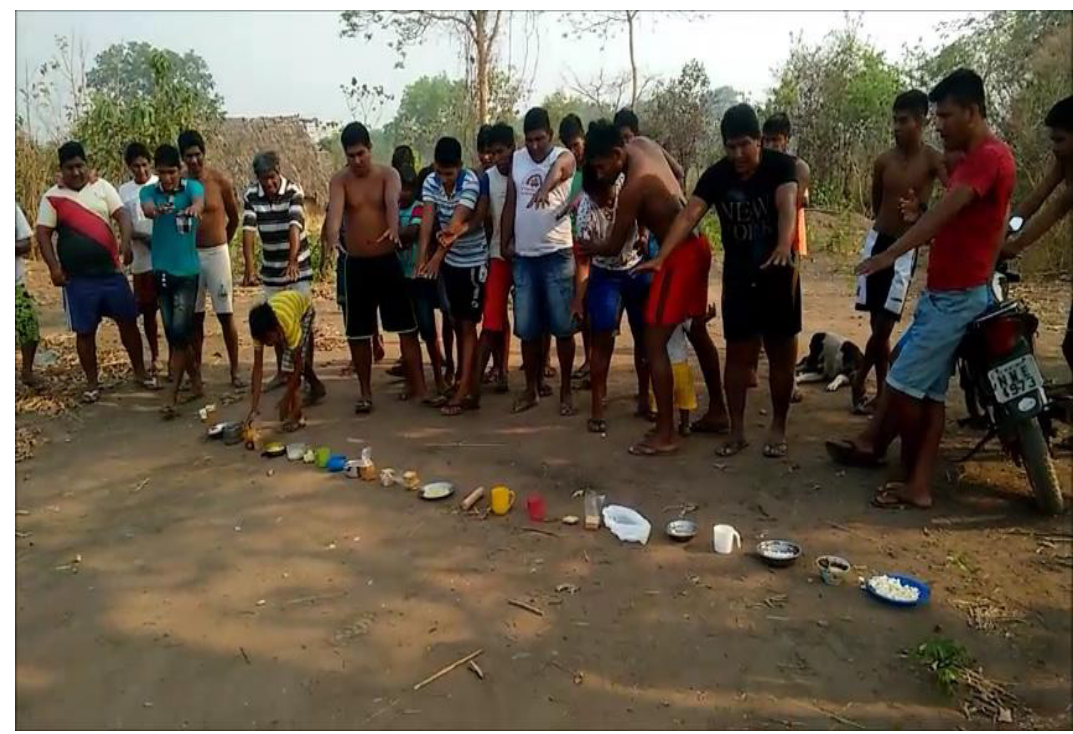

Fonte: Kaorewygoo Tapirapé.

Foto 2 - Kaki tahè jyrè mahãdù bùtùna-my riu-my rirahùra ixy riudỹỹnmy, ahu ijyki tamy rohula renyra-u.

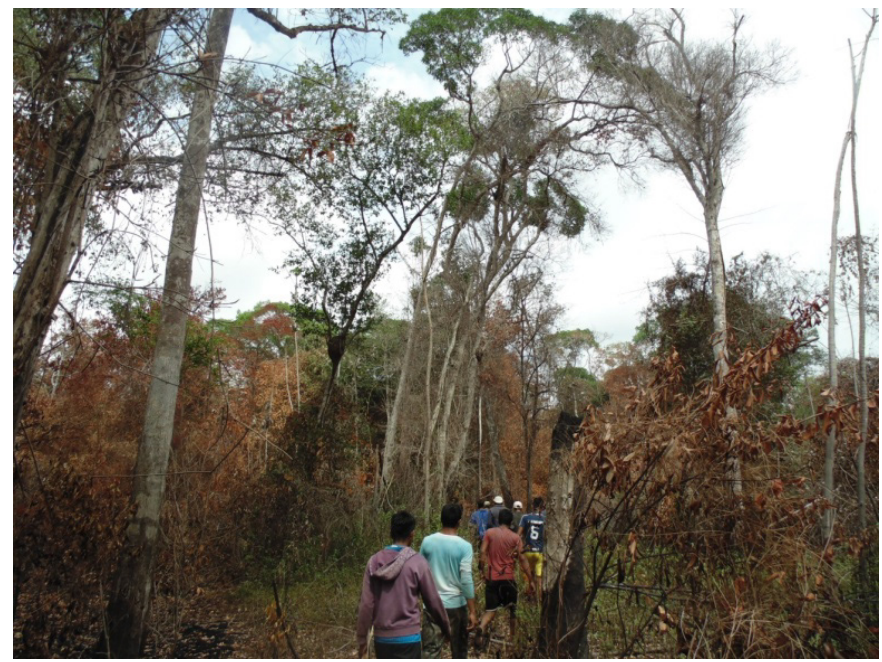

Fonte: Kaorewygoo Tapirapé. 
Foto 3 - Ahu ijyki tahè jyrè mahãdù widèè rarybè rènyreri wii riwirarèarènykremy,ibòò-ò irarù-ò ijo rèakre-my.

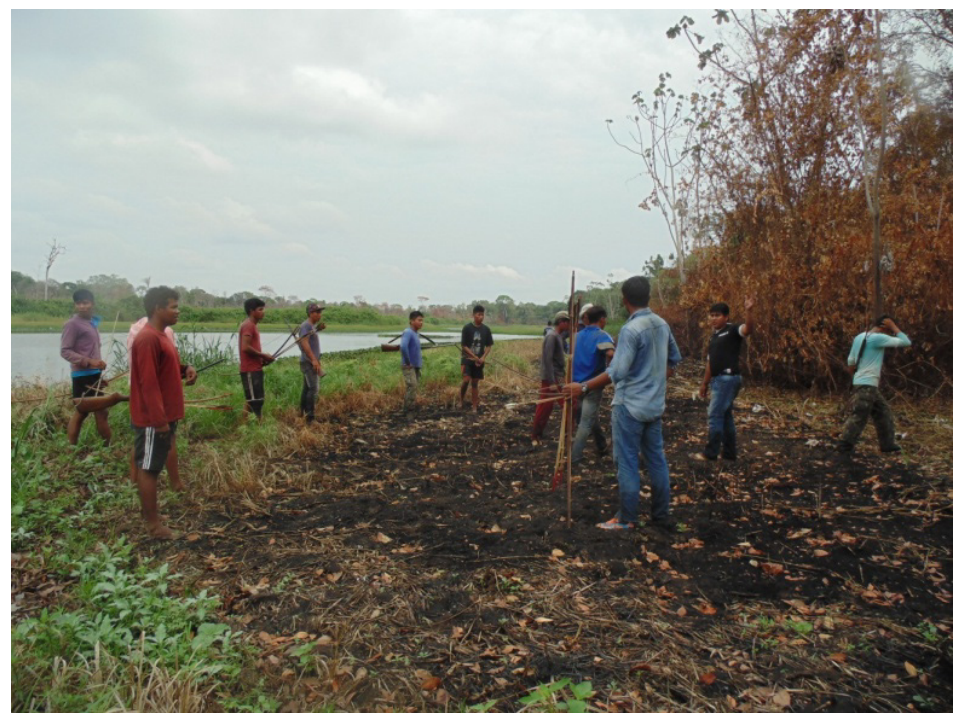

Fonte: Kaorewygoo Tapirapé.

Foto 4 - Bdèru wòò risõre kia tyaki tahè kumairu, tei, hãwãlari, hãdòri, mylauri kia boho ixy-ò roholarènyreri.

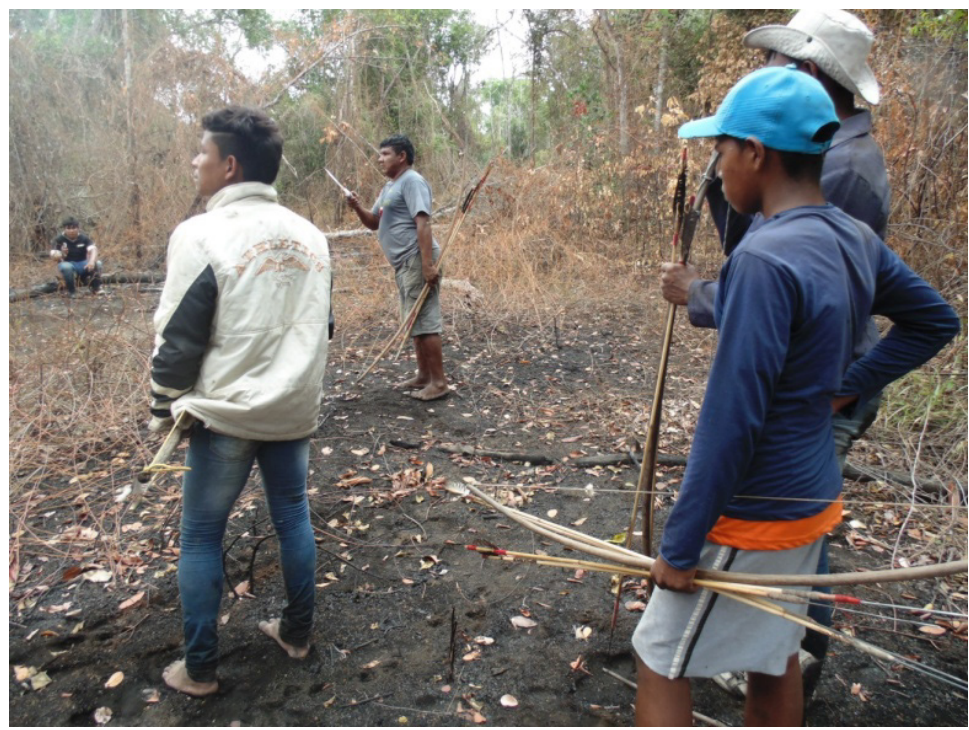

Fonte: Kaorewygoo Tapirapé. 
Foto 5 - Kaki tahè bdèro-di ix $\tilde{y}$-di retyy-my rirahurèri, kiemy juhu kawse idi rirahùmyhỹre bdèro-di.

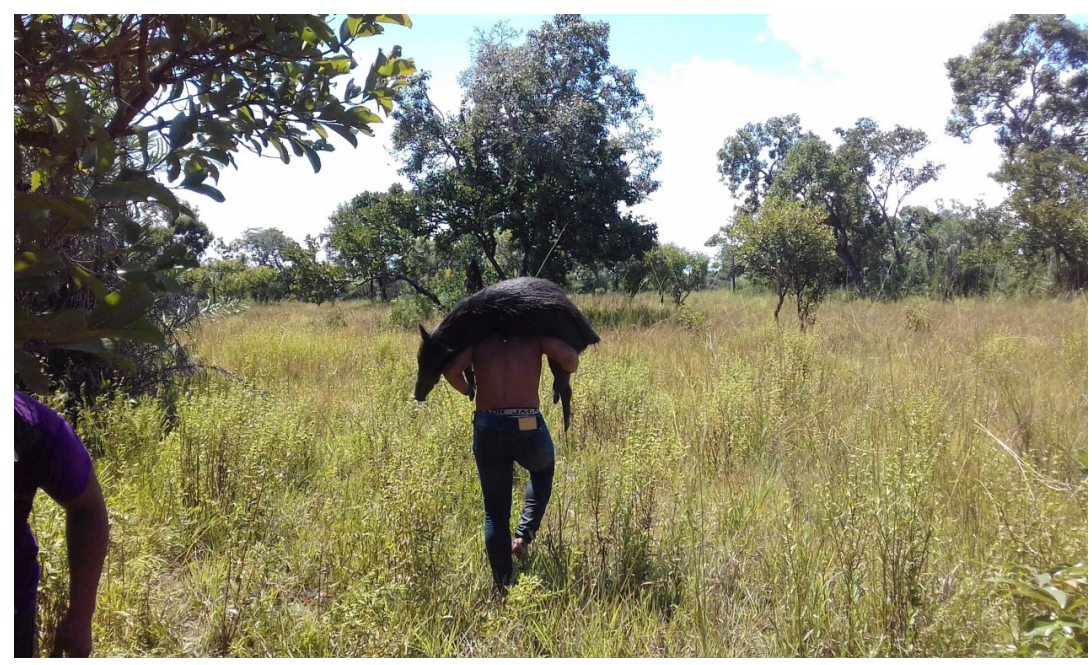

Fonte: Kaorewygoo Tapirapé.

Foto 6 - Kawse tahè reuròmyhỹre ix $\tilde{y}$ ryrò-di bùtùnahãkỹmy, iò tarkihè itxi ijradù tamy rèamyhỹre rirbunymyhỹre inatxi dè inataò aobo irbi, tarkihè idi namyhède worysy.

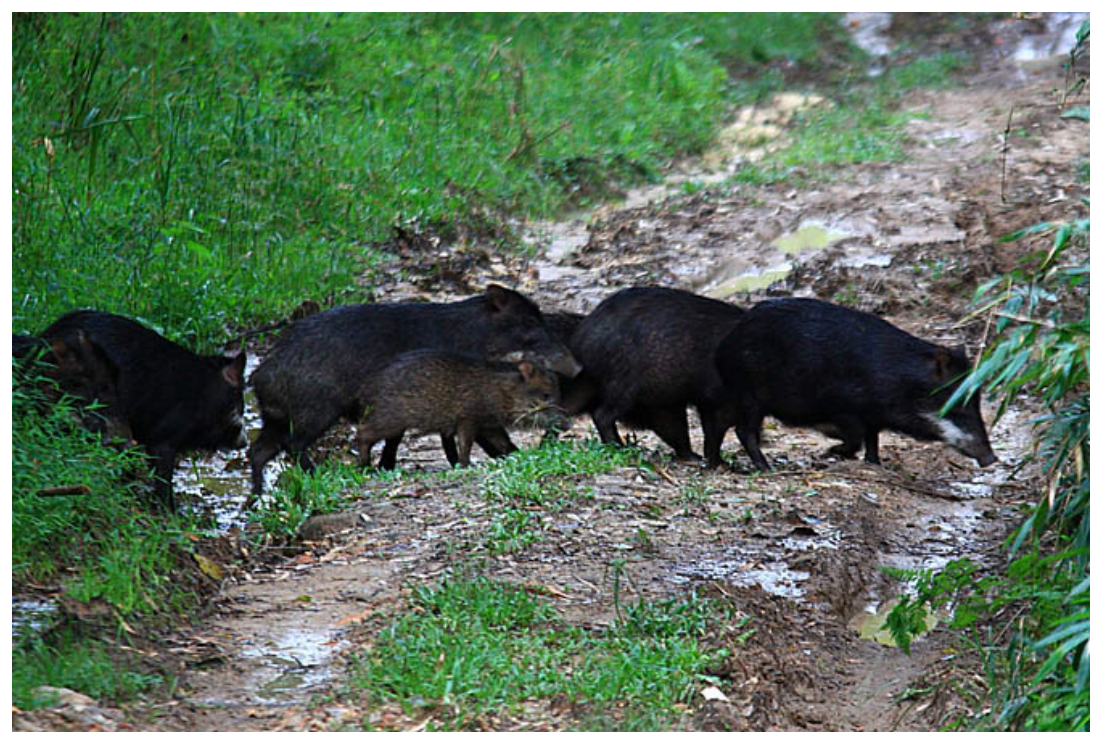

Fonte: Kaorewygoo Tapirapé. 
Foto 7 - Juhu bde-u rkihè iny mahãdù ynyra-my raxyny-my rỹiramyhỹ taiki-my tahè ix $\tilde{y}$ rubèhèmyhỹre, iòlè tamy rèamyhỹrènyre irutaòle-my.

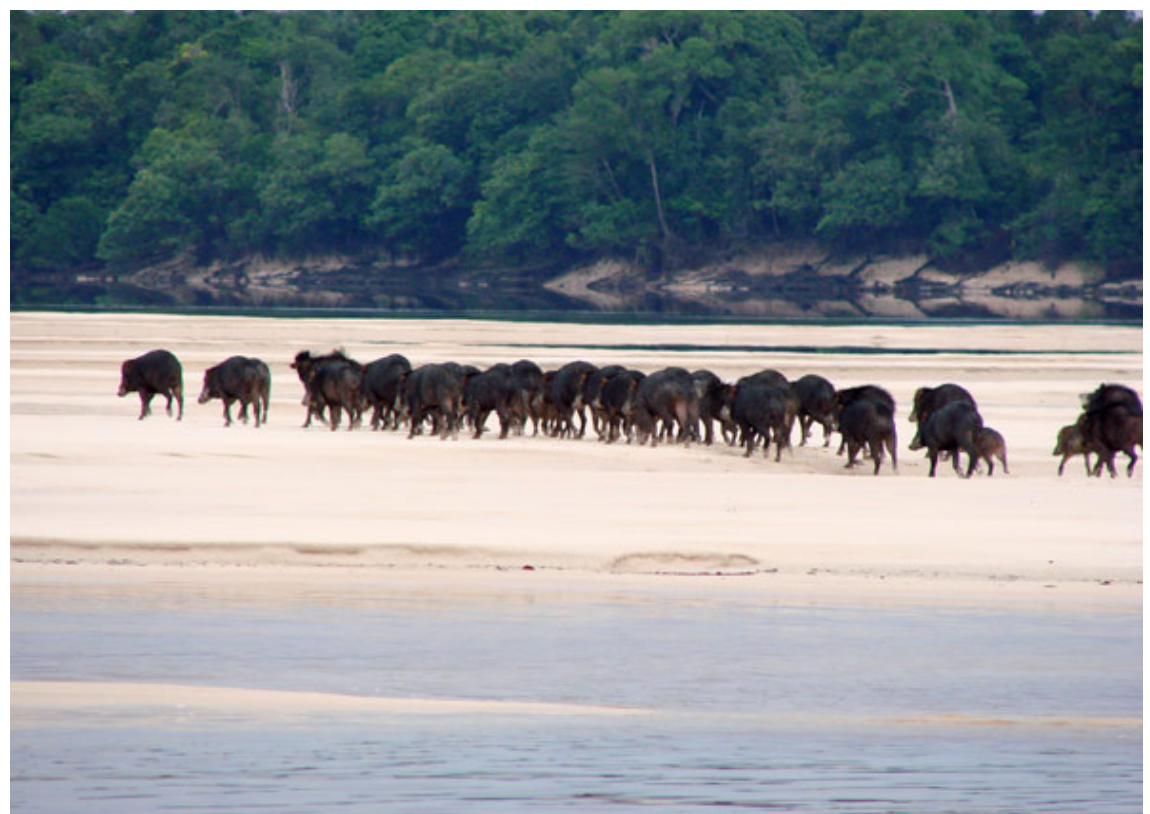

Fonte: Kaorewygoo Tapirapé.

Recebido para publicação em março de 2018. Aceito para publicação em maio de 2018. 